\title{
Monodispersed $\mathrm{CsPb}_{2} \mathrm{Br}_{5} @ \mathrm{SiO}_{2}$ Core-Shell Nanoparticles as Luminescent Labels for Biosensing
}

\author{
Cynthia Collantes, Victoria González Pedro,* María-José Bañuls, and Ángel Maquieira
}

Cite This: ACS Appl. Nano Mater. 2021, 4, 2011-2018

Read Online

ABSTRACT: Despite the rising advances in the field of metal halide perovskite nanocrystals (NCs), the exploitation of such nanoparticles as luminescent labels for ex vivo imaging and biosensing is still unclear and in the early stages of investigation. One of the major challenges toward the implementation of metal halide perovskite NCs in biosensing applications is to produce monodispersed nanoparticles with desired surface characteristics and compatible with aqueous environments. Here, we report the synthesis of monodispersed spherical $\mathrm{CsPb}_{2} \mathrm{Br}_{5} @ \mathrm{SiO}_{2}$ core-shell nanoparticles by post-synthetic chemical transformation of $3 \mathrm{D}$ $\mathrm{CsPbBr}_{3} \mathrm{NCs}$ in the presence of tetraethyl orthosilicate and a critical water/ammonia ratio. This method involves an ammonia-

(a)

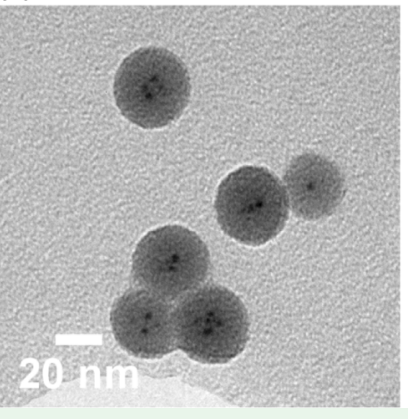

(b)

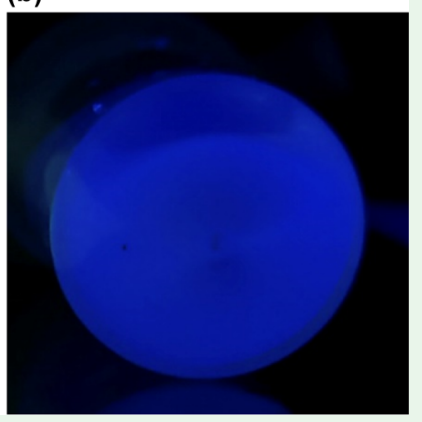
mediated and ammonia-induced "top-down" transformation of assynthesized $3 \mathrm{D} \mathrm{CsPbBr} 3$ NCs to smaller $\mathrm{CsPb} 2 \mathrm{Br} 5$ nanoclusters (ca. 2-3 nm), which trigger a seed-mediated silica growth, yielding monodispersed spherical blue luminescent $\left(\lambda_{\text {emission }}=432 \mathrm{~nm}\right) \mathrm{CsPb}_{2} \mathrm{Br}_{5} @ \mathrm{SiO}_{2}$ perovskite nanoparticles. By adjusting the reaction conditions, core-shell nanoparticles of a $36.1 \pm 4.5 \mathrm{~nm}$ diameter, which preserve their optical properties in water, were obtained. Besides that, the viability of the developed nanoparticles as a luminescent label for biosensing has been proven by specific biorecognition of the IgG protein in a direct immunoassay. Our work sheds light on the chemical processes and transformations involved in the silica nucleation mechanism in the presence of perovskite nanoparticles and opens the way for the future rational design of the next generation of semiconductor NC luminescent biological labels.

KEYWORDS: cesium lead bromide nanocrystals, silica growth, $\mathrm{CsP}_{2} \mathrm{Br}_{5} @ \mathrm{SiO}_{2}$ core-shell nanoparticles, monodisperse, stability, luminescent label, protein detection

\section{INTRODUCTION}

Metal halide perovskite (MHP) nanocrystals (NCs) have attracted great interest owing to their excellent optical features such as composition-tunable band gap, easy synthesis, and high photoluminescence quantum yields (PLQYs $=50-90 \%)$ and have demonstrated superior properties in photovoltaics and light emission devices. ${ }^{1}$ Besides that, they also possess higherorder nonlinear optical properties, ${ }^{2-4}$ with reported absorption cross sections for up to five photons, ${ }^{4}$ which make them a promising luminophore with a bright future in many areas of modern biology such as labels and contrast agents for in vitro bioimaging and biosensing (i.e., immunostaining, DNA and protein microarraying, and flow-cytometry-based diagnosis) and for super-resolution imaging applications going beyond the optical diffraction limits.

Toward their implementation as luminescent probes, is necessary NCs confinement inside suitable protective materials which accomplishes a dual role: prevent their decomposition in a water medium and endow their chemical functionalization with appropriated groups. In this framework, the use of alcoxysilanes is a smart and very straightforward route for encapsulating MHP
NPs because silica is chemically stable and transparent in the whole visible region, protects materials against moistureinduced damage, and possesses facile conjugation with different functional groups to enable further functionalization. ${ }^{6}$

Accordingly, some successful examples have been reported. $^{7-20}$ However, most efforts using these conventional $\mathrm{SiO}_{2}$ coating methods for perovskite NPs failed because perovskite NCs are too sensitive to conditions of Stöber methodology (i.e., the presence of water in a basic medium), which leads to their dissolution. ${ }^{21}$ This involves working in soft conditions, resulting in random alkoxysilane polymerization processes. Thus, until date, most of the reported methods were only successful in dealing with an ensemble of MHP NCs and the products were macroscale particles. In this sense, gaining

Received: December 15, 2020

Accepted: February 3, 2021

Published: February 12, 2021 
(a)

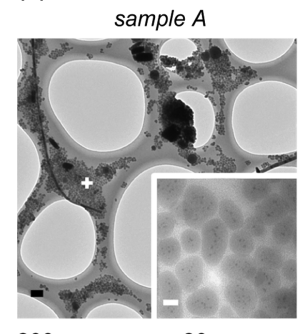

$200 \mathrm{~nm}$ (b)

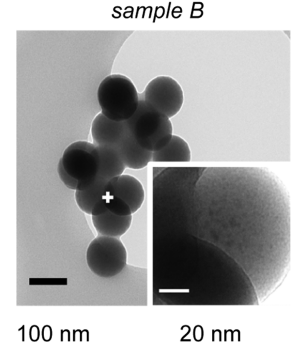

(c)

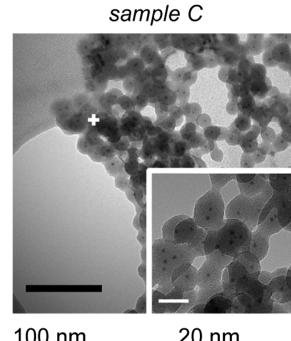

(d)

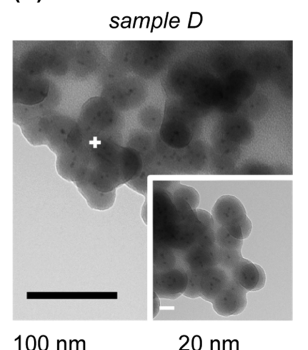

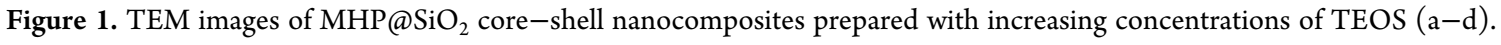

insight into the silica growth mechanism on MHP and the development of a methodology to synthesize monodispersed $\mathrm{MHP} @ \mathrm{SiO}_{2}$ are very challenging tasks and only few works have reported their encapsulation into an oxide matrix at a singleparticle level. Some examples have successfully achieved this goal by adaptation of sol-gel processes. For instance, Cheng et al. reported room-temperature crystal growth of $\mathrm{Cs} \mathrm{PbBr}_{3}$ inside pre-synthesized amine-functionalized silica micelles. ${ }^{19} \mathrm{Ding}$ et al. $^{20}$ developed a reverse microemulsion methodology employing $n$-hexylalcohol: cyclohexane mixture, ammonium hydroxide, and triton $x-100$ as surfactant, and $\mathrm{Hu}$ et al. ${ }^{17}$ combined a watertriggered transformation process of $\mathrm{Cs}_{4} \mathrm{PbBr}_{6} \mathrm{NCs}$ to $\mathrm{CsPbr}_{3}$ and a sol-gel method for preparing $\mathrm{CsPbBr}_{3} @ \mathrm{SiO}_{2}$ and $\mathrm{CsPbr}_{3} @ \mathrm{Ta}_{2} \mathrm{O}_{5}$ Janus NPs. On the other hand, other researchers have focused on alternative methods for $\mathrm{SiO}_{2}$ coating. Among them, Huang et al. ${ }^{11}$ report in situ growth into hollow siliceous nanospheres and Song et al. ${ }^{9}$ synthesized perovskite core-shell nanocubes via a modified hot injection method, wherein a certain part of oleylamine was substituted with (3-aminopropyl)triethoxysilane (APTES).

Here, we report a novel and effective approach for preparation of monodispersed silica NPs containing MHP NCs based on the controlled chemical transformation of $3 \mathrm{D} \mathrm{CsPbBr}$ in the presence of tetraethyl orthosilicate and a critical water/ammonia ratio. Under these reaction conditions, $3 \mathrm{D} \mathrm{CsPbBr}_{3} \mathrm{NCs}$ transform into $\mathrm{CsPb}_{2} \mathrm{Br}_{5}$ nanoclusters of $2-3 \mathrm{~nm}$, which act as silica nucleation seeds rendering monodispersed spherical blue luminescent $\mathrm{CsPb}_{2} \mathrm{Br}_{5} @ \mathrm{SiO}_{2}$ perovskite nanoparticles (NPs). The facile and effective functionalization of the developed nanoparticles with vinyl and amine groups by easy postsynthetic treatment was confirmed by attenuated total reflectance Fourier-transform infrared spectroscopy (ATRFTIR).

Besides that, in this study, the developed NCs were used as a fluorescent label in immunoassays for specific detection of bovine serum albumin (BSA). For that purpose, we developed the perovskite NCs labeled antiBSA antibodies, which were applied to the specific biorecognition of proteins. BSA was used as an example of a target protein.

\section{RESULTS AND DISCUSSION}

In our methodology, colloidal CsPbBr 3 NCs $(11.04 \pm 2.19 \mathrm{~nm}$ edge length, PLQY 90\%) with an emission maximum at 515 $\mathrm{nm}$ were synthesized according to published protocols. ${ }^{22}$ (See Figure $\mathrm{S} 1$ in the Supporting Information for more details). Then, for synthesizing silica-overcoated MHP NCs, $0.5 \mathrm{~mL}$ of $\mathrm{CsPbr}_{3}$ (40 nM in toluene) was treated with $2 \mu \mathrm{L}$ of concentrated aqueous ammonia $(25 \% \mathrm{w} / \mathrm{w})$ and different tetraethylorthosilicate (TEOS) equivalents. The mixture was incubated overnight under stirring $(300 \mathrm{rpm})$ and the resulting
$\mathrm{MHP} @ \mathrm{SiO}_{2}$ core-shell NPs were collected by centrifugation and dispersed in toluene. The content of $\mathrm{NH}_{4}{ }^{+}$is critical in the shell growth and formation of isolated core-shell nanoparticles because ammonia serves as a basic catalyst for the hydrolysis/ condensation process of the silica precursor, reduces the amount of energy to star nucleation, and also has a role in protecting the newly formed silica particles from aggregation. ${ }^{23}$ In our work, we optimized the ammonia content to $2 \mu \mathrm{L}$ of a concentrated base. Larger volumes produce a degradation of perovskite nanoparticles, while lower volumes lead to a random nucleation of TEOS monomers in a macroscale assemble.

The concentration of TEOS and water play a key role in the spatially controlled deposition of $\mathrm{SiO}_{2}$. First, to evaluate the influence of TEOS concentration, we analyzed the morphology of the silica shell (grown on the NPs surface) at different alkoxysilane equivalents and a constant volume of water $(2 \mu \mathrm{L}$ of concentrated aqueous ammonia $25 \% \mathrm{w} / \mathrm{w}$ ). Structural features and reactant concentrations for the resulting $\mathrm{SiO}_{2}$-overcoated perovskite nanocomposites are summarized in Table S1.

Figure 1 shows the transmission electron microscopy (TEM) images of samples A-D treated with different amounts of TEOS $(60,310,480$, and $960 \mu \mathrm{L}$, respectively). It is remarkable that all TEM images present $2-3 \mathrm{~nm}$ perovskite NCs embedded into silica nanoparticles with different sizes and morphologies. Sample A shows MHP NCs encapsulated into ellipsoidal silica nanoparticles with a major diameter and a minor diameter of $35.5 \pm 8.4$ and $20.7 \pm 3.4 \mathrm{~nm}$, respectively. In addition, it also exhibits a small population of nanoparticles $(162 \pm 38 \mathrm{~nm}$ averaged diameter) and nanowires ( $56 \pm 14 \mathrm{~nm}$-thick), which could be attributed to the ripening mechanism and rearrangement of original $\mathrm{CsPbBr}_{3} \mathrm{NCs}$ into larger structures. ${ }^{24-26}$ In sample $\mathrm{B}$, the resulting nanomaterial evolved into the formation of spherical silica nanoparticles of $101.4 \pm 6.5 \mathrm{~nm}$ (average diameter). However, samples $\mathrm{C}$ and $\mathrm{D}$ render a silica nanoparticle cross-linked network with an average diameter of $19.7 \pm 2.4$ and $33.8 \pm 4.0 \mathrm{~nm}$, respectively.

Remarkably, larger amounts of TEOS volumes led to the formation of macroscale ensembles (Figure S2a). This fact is attributed to the large amount of silane, which produces random polymerization of alkoxysilane monomers in macroscale composites. These data are similar to those obtained for the control sample in the absence of basic aqueous equivalents (Figure S2b), which points out the key role of the aqueous ammonia in the nanoparticle nucleation mechanisms.

Another critical parameter controlling the silica shell growth is the water amount. To test the influence of water in shell morphology, $0.5 \mathrm{~mL}$ of $\mathrm{CsPbBr}_{3} \mathrm{NCs}$ was treated with $310 \mu \mathrm{L}$ of TEOS, $2 \mu \mathrm{L}$ of aqueous ammonia, and increasing water volumes. As perovskite particles decomposed in the excess of basic aqueous medium, a fractioned dropped method was adopted 
(a)

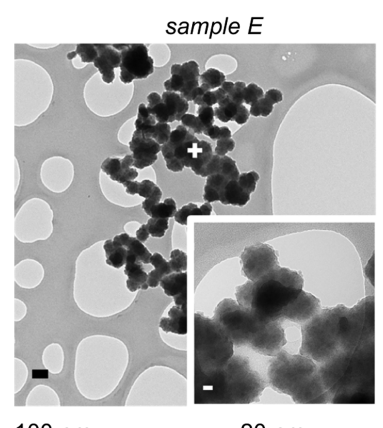

(b)

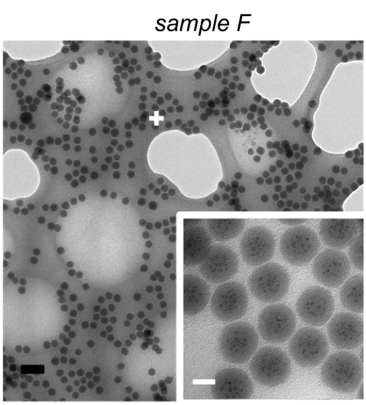

$100 \mathrm{~nm}$ (c)

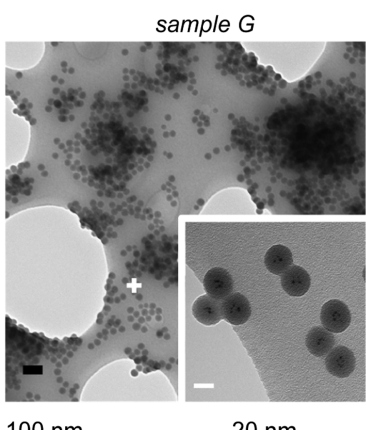

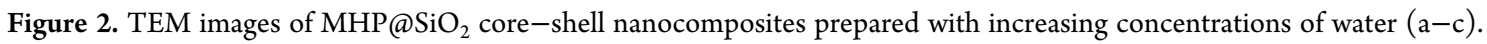

with the aim to slow down this process, in which TEOS and ammonia dissolved in water were added with a flow rate of 0.74

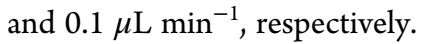

As shown in Figure 2 and Table S1, monodispersed coreshell nanoparticles with an average diameter size in the range of $30 \mathrm{~nm}$ were achieved for samples $\mathrm{F}$ and $\mathrm{G}$ treated with 28 and 48 $\mu \mathrm{L}$ of water, respectively. Larger volumes of water produce hydration of $\mathrm{CsPbBr}_{3} \mathrm{NCs}$, triggering their decomposition up to their ionic precursors, ${ }^{27-29}$ therefore leading to the formation of core-free silica nanoparticles (Figure S3). The differences observed between samples A and E prepared with the same reagent concentration arise from the dropped fractioned methodology adapted for preparation of sample E. These data highlight the key importance of water in obtaining highly monodispersed nanoparticles. In addition, the experiment was repeated, but this time increasing the final volume of the TEOS reagent to 480 and $960 \mu \mathrm{L}$. In both cases, the resulting products led to silica agglomeration (see Figure S4, Supporting Information).

In order to understand these observations, we first need to consider the chemical processes involved in the nucleation of silica: hydrolysis and polycondensation of TEOS. With less equivalents of water, most of the TEOS monomers will remain unhydrolyzed; thus, the process is driven by the spontaneous polycondensation of TEOS alkoxide species, which leads to a random nucleation mechanism, resulting in size and shape variability of silica nanoparticles (as shown in samples A to E). With 28 and $48 \mu \mathrm{L}$ of water, 30 and $50 \%$ of TEOS monomers are partially hydrolyzed. Since these monomers are deprotonated due to the high $\mathrm{pH}$ value, in the presence of MHP NCs, hydrolyzed TEOS monomers are attracted toward $\mathrm{Pb}$ atoms and hence replace the original capping molecules, leading to a localized growth of uniform-sized silica shells.

The structural and optical features of monodisperse coreshell nanoparticles from sample $G$ are summarized in Figure 3a,b and Table S2. The as-synthesized nanoparticles exhibit a blue luminescent peak centered at $432 \mathrm{~nm}$ with a PLQY of $~ 5 \%$ and an average particle diameter of $36.1 \pm 4.5 \mathrm{~nm}$.

Paying attention to the existing literature, although there is a variety of works which agree on structure and composition of $\mathrm{CsPb}_{2} \mathrm{Br}_{5} \mathrm{NCs}$, they greatly diverge on the interpretation of their intrinsic optical properties, which currently is a subject under debate. For instance, there are works which attribute not noticeable photoluminescence (PL) to $\mathrm{CsPb}_{2} \mathrm{Br}_{5}$ NCs. ${ }^{30,31}$ Other works describe $\mathrm{CsPb}_{2} \mathrm{Br}_{5}$ clusters with an $\mathrm{UV}$ emission edge at $380 \mathrm{~nm}^{32}$ and a dual emission behavior ( $\sim 60$ and 520 $\mathrm{nm}$ ) with an emission quantum yield of $4 \% .{ }^{33}$ Besides that, other reports on $\mathrm{CsPb}_{2} \mathrm{Br}_{5}$ nanoplatelets and microplates suggest a (a)
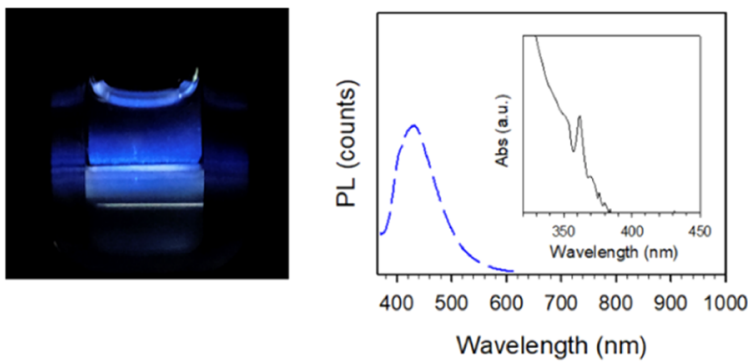

(b)

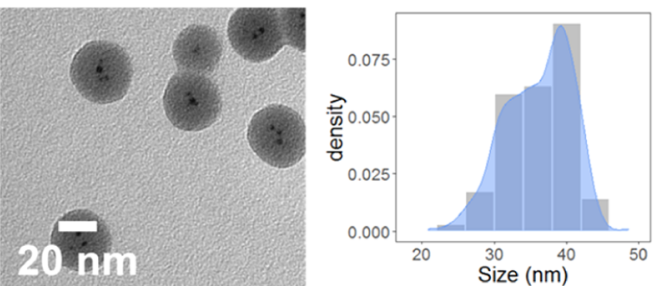

(c)

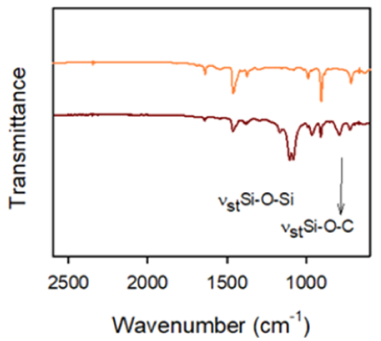

Figure 3. (a) Image of blue-emitting core-shell $\mathrm{MHP} @ \mathrm{SiO}_{2} \mathrm{NCs}$ at $\lambda_{\text {exc }}=254 \mathrm{~nm}$. Absorbance (black line) and emission spectra (blue line) of $\mathrm{MHP} @ \mathrm{SiO}_{2} \mathrm{NCs}$. The PL spectrum was recorded at an excitation wavelength $\lambda_{\text {exc }}=355 \mathrm{~nm}$. (b) TEM image of monodispersed coreshell nanoparticles and particle characterization. (c) ATR-FTIR

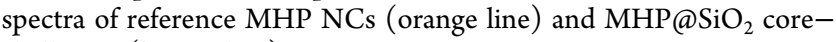
shell NCs (brown line).

material with strong blue to green tunable emission properties. ${ }^{34-36}$ Unfortunately, the exact electronic structure and PL mechanism in $\mathrm{CsPb}_{2} \mathrm{Br}_{5}$ is not completely known and the emission properties have yet to be fully established because of the existence of direct and indirect transitions. ${ }^{30,37}$ Although this topic is worthy to be studied, we considered that is beyond the scope of this manuscript.

The ATR-FTIR spectra of bare and core-shell NCs are shown in Figure 3c. The peak at $\sim 1050 \mathrm{~cm}^{-1}$ corresponds to the asymmetric stretching of $\mathrm{Si}-\mathrm{O}-\mathrm{Si}$ linkage and confirms the formation of a cross-linked siloxane network surrounding the 


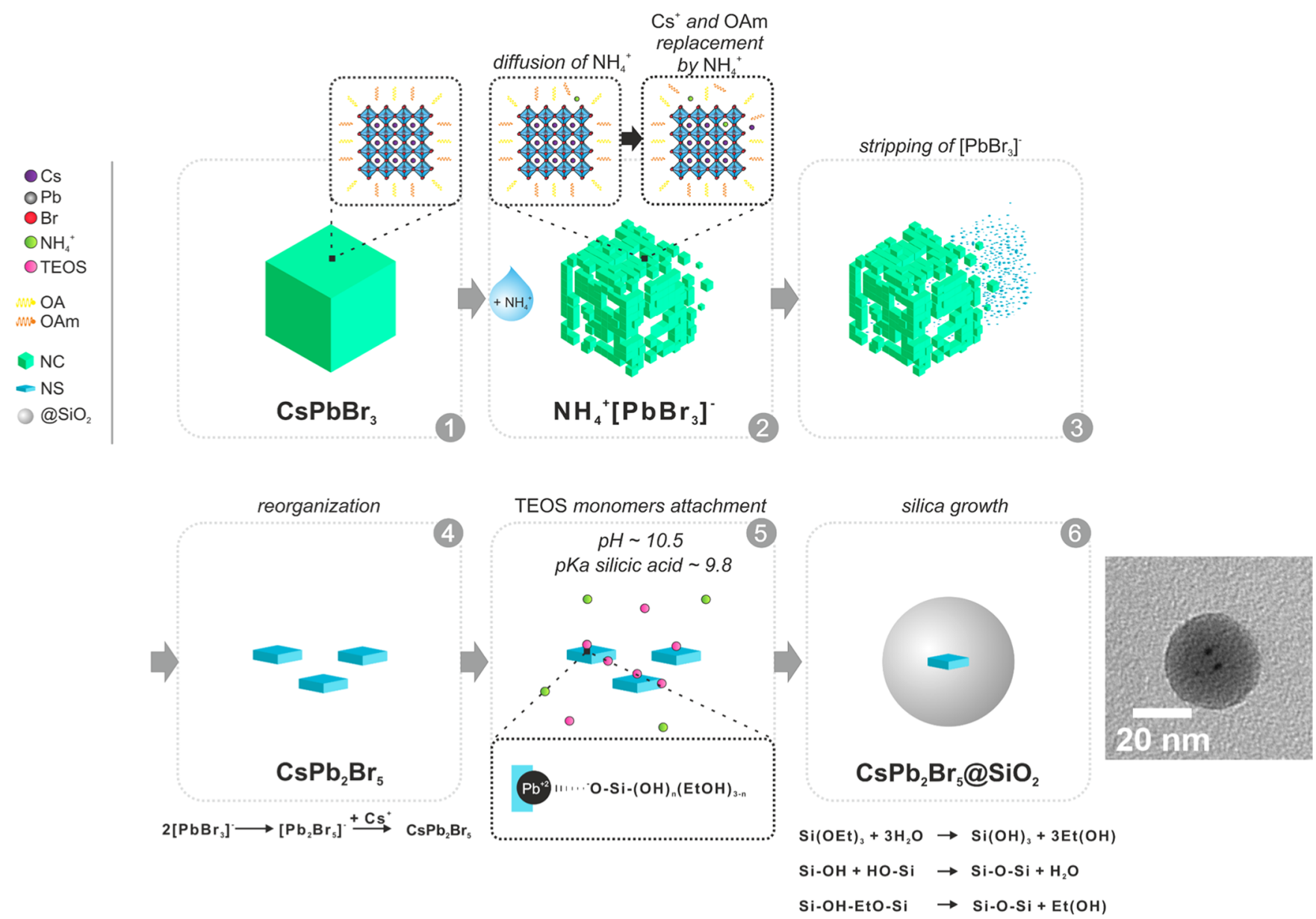

Figure 4. Schematic representation of the formation mechanism of $\mathrm{CsPb}_{2} \mathrm{Br}_{5}$ silica-coated core-shell $\mathrm{NPs}$ from $\mathrm{CsPbBr} \mathrm{NCs}_{3}$. The proposed mechanism involves diffusion and intercalation of $\mathrm{NH}_{4}^{+}$cations into a $\mathrm{CsPbBr}$ native structure (step 1-2), exfoliation of $\left[\mathrm{PbBr}_{3}\right]^{-}$smaller nanoclusters through a toluene-water interface (step 3), reorganization into $\mathrm{CsPb}_{2} \mathrm{Br}_{5} \mathrm{NCs}$ (step 4), and seed-mediated growth of uniform silica shells (steps 5 and 6).

perovskite nanoparticle. There is also a peak at $790 \mathrm{~cm}^{-1}$ that corresponds to the $\mathrm{Si}-\mathrm{O}-\mathrm{C}$ symmetric stretching of the unreacted alkoxysilane groups. EDX-TEM elemental analysis (Figure S5) reveals that the atomic $\mathrm{Si} / \mathrm{O}$ ratio of the shell was $1: 2$, close to the theoretical value.

In order to identify the nanoparticle core composition and determine the $\mathrm{Cs}, \mathrm{Pb}$, and $\mathrm{Br}$ stoichiometric element relations, inductively coupled plasma mass spectrometry (ICP-MS) measurements were carried out. The elemental analysis results are shown in Table S3 in the Supporting Information. Paying attention to the $\mathrm{Cs}$-to- $\mathrm{Pb}$ obtained ratio (0.56) reveals the formation of $\mathrm{CsPb}_{2} \mathrm{Br}_{5}$ perovskite-related NCs. Concerning the large $\mathrm{Br}$-to- $\mathrm{Pb}$ molar ratios registered, they can be attributed to the technical difficulty for determining bromide species by ICPMS, which typically interferes with the Ar dimer of plasmogen gas. $^{38-40}$

On the basis of the results discussed above, we suggest that the transformation process is probably controlled by the intercalation of ammonia cations, the replacement of Cs positions, and the variation of the coordination number of $\mathrm{Pb}^{2+}$ in water. $\mathrm{A}$ plausible schematic mechanism of the crystal structure evolution is shown in Figure 4 and eqs 1 and 2, and the related processes can be described as follows. The first step is the diffusion of ammonia in the perovskite network; then, $\mathrm{Cs}^{+}$cations in the perovskite material are replaced by $\mathrm{NH}_{4}^{+}$to form $\mathrm{NH}_{4} \mathrm{PbBr}_{3}$, resulting in distinct changes in the crystalline structure. This fact, along with the replacement of oleylamine by ammonia molecules, induces exfoliation of $x \mathrm{NH}_{4}^{+}\left[\mathrm{PbBr}_{3}\right]^{-}$smaller nanoclusters through the toluene-water interface, which will interact to form $\mathrm{CsPb}_{2} \mathrm{Br}_{5} \mathrm{NCs}$ (see eqs 1 and 2). It is noteworthy that a recent work of Liu et al. reports the change of the coordination number of $\mathrm{Pb}^{2+}$ from six to eight and transformation to $\mathrm{CsPb}_{2} \mathrm{Br}_{5}$ in a humid environment. ${ }^{41}$ In another work, Balakrishnan and $\mathrm{Kamat}^{33}$ reported phase transformation under dodecyldimethylammonium bromide treatment. Although in this latter phase, transition only occurs for long alkyl chain ammonium cations, our work differs because of the combined effect of water and ammonium. Then, deprotonated TEOS monomers can attach to lead dangling bonds of the released NCs, triggering a seed-mediated growth of uniform silica overcoating surrounding the perovskite NCs and blocking the formation of larger $\mathrm{CsPb}_{2} \mathrm{Br}_{5}$ nanosheets.

$$
\begin{aligned}
& n \mathrm{CsPbBr}_{3}+x \mathrm{NH}_{4}^{+} \\
& \quad \rightarrow(n-x) \mathrm{CsPbBr}_{3}+x \mathrm{NH}_{4}^{+}\left[\mathrm{PbBr}_{3}\right]^{-}+x \mathrm{Cs}^{+} \\
& 2 x \mathrm{NH}_{4}^{+}\left[\mathrm{PbBr}_{3}\right]^{-}+x \mathrm{Cs}^{+} \\
& \quad \rightarrow x \mathrm{CsPb}_{2} \mathrm{Br}_{5}+x \mathrm{NH}_{4}^{+} \mathrm{Br}^{-}+x \mathrm{NH}_{4}^{+}
\end{aligned}
$$

In addition, high-resolution transmission electron microscopy (HR-TEM) measurements were also carried out with the aim to 
(a)
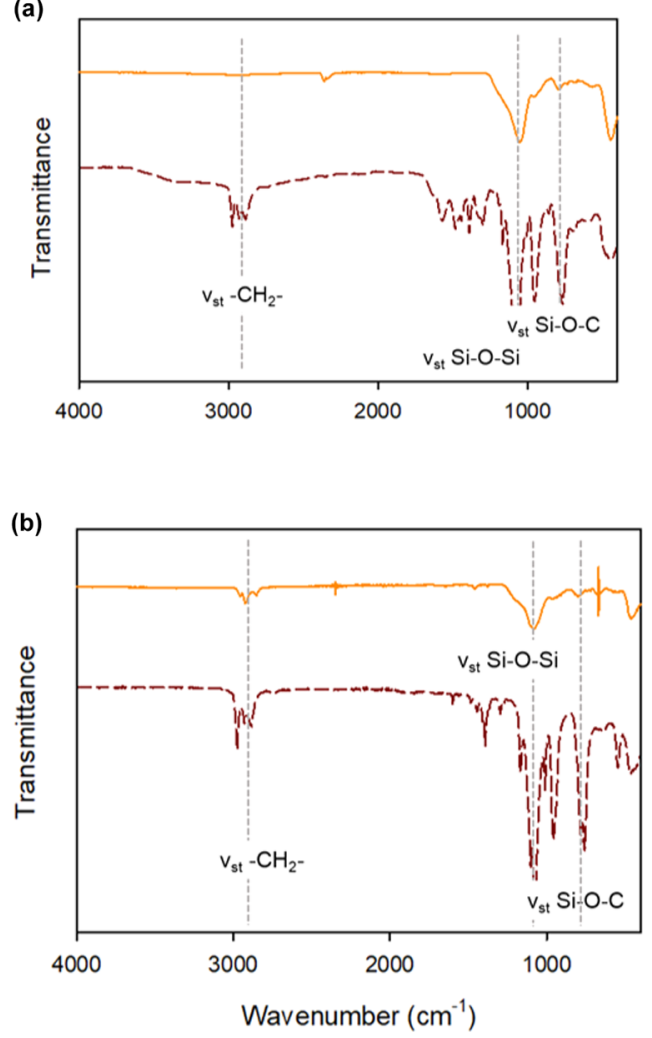
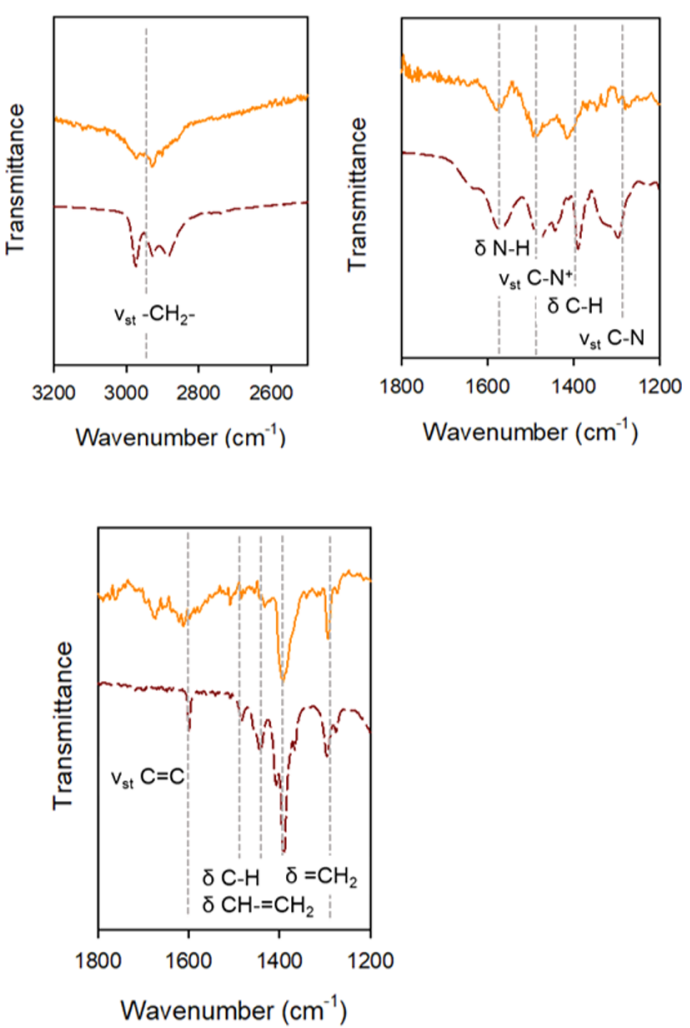

Figure 5. FTIR spectra of reference alcoxysilanes (brown line) and $\mathrm{MHP}_{2} @ \mathrm{SiO}_{2}$ core-shell NCs (orange line) functionalized with (a) 3aminopropyltriethoxysilane and (b) vinyltriethoxysilane.

(a)

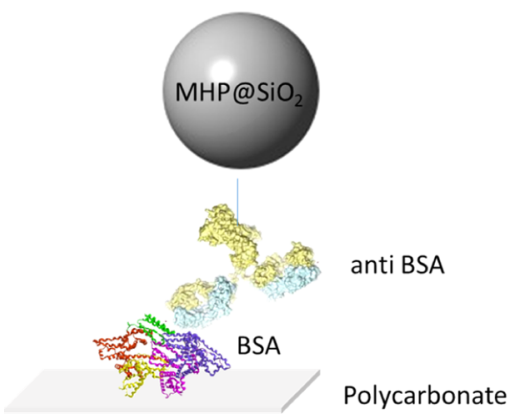

(b)

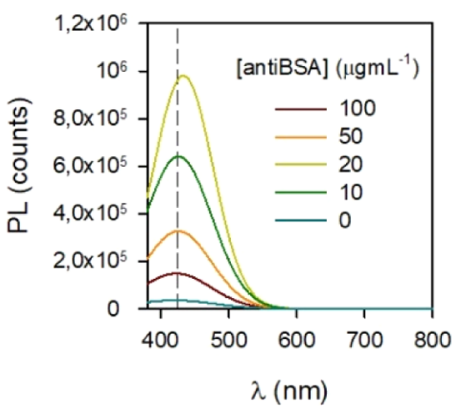

(c)

[antiBSA]
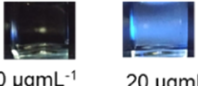

$20 \mu \mathrm{gmL}^{-}$

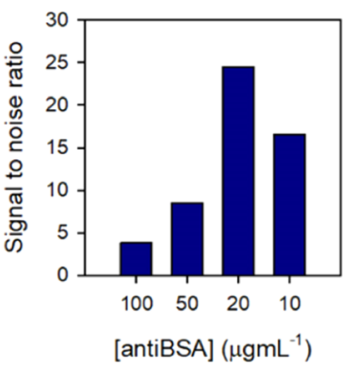

Figure 6. (a) Direct immunoassay scheme. (b,c) PL response and signal to noise ratio obtained for different NP/antibody ratios. The top image in (c) depicts a picture of samples treated with 0 and $20 \mu \mathrm{g} \mathrm{mL}{ }^{-1}$ of anti-BSA.

unveil the nature of perovskite NCs. However, it has not been possible to determine the crystal lattice using HR-TEM either because of the small proportion of the crystal lattice or because of the considerable $\mathrm{SiO}_{2}$ shell thickness, which make its detection difficult.

In an additional experiment, increasing the reaction time from 24 to $48 \mathrm{~h}$, the formation of agglomerated perovskite core-shell NCs was observed. Among them, there was a small population of silica nanoparticles which contain larger perovskite NCs $(\sim 7.5$ $\mathrm{nm}$ ) embedded inside. Probably, the presence of these NCs is due to the formation of larger NCs from etched $\mathrm{Cs}^{+}$and $\mathrm{NH}_{4}^{+}\left[\mathrm{PbBr}_{3}\right]^{-}$at longer reaction times, which are then encapsulated inside silica shells. The HR-TEM image of these NC cores reveals that the interfringe distance is about $0.3 \mathrm{~nm}$ (Figure S6), corresponding to the (220) lattice plane of the crystal, ${ }^{33,36}$ thus confirming the formation of $\mathrm{CsPb}_{2} \mathrm{Br}_{5} \mathrm{NCs}$.
The absence of core-free silica nanoparticles in sample G could be explained according to LaMer theory, ${ }^{42,43}$ which claims that the energy barrier governing the heterogeneous nucleation is lower than that governing the homogeneous nucleation, and empty nanoparticles only form when the concentration of TEOS monomers surpasses the homogeneous nucleation threshold. In this sense, our fractionated drop method can always meet the above-mentioned conditions because fresh TEOS is added after the previous TEOS is mainly consumed.

The stability of synthesized core-shell nanoparticles has been confirmed by dispersing $5 \mathrm{mg}$ of the collected NCs in $5 \mathrm{~mL}$ of water and sonicating for $2 \mathrm{~min}$ (Sonorex Super, Bandelin Co., Germany) with a frequency of 50/60 Hz. As shown in Figure S7, the blue emission of the core-shell nanostructures could still be observed clearly after $4320 \mathrm{~min}$ (3 days). 
The easy and effective functionalization of the developed nanoparticles with amine- and vinyl-functional groups was demonstrated by additional post-synthetic treatment with alkoxysilanes bearing the respective terminations and confirmed by ATR-FTIR spectroscopy (Figure 5). NCs functionalized with APTES present characteristic stretching of $\mathrm{Si}-\mathrm{O}-\mathrm{Si}(1050$ $\left.\mathrm{cm}^{-1}\right)$ and $\mathrm{Si}-\mathrm{O}-\mathrm{C}\left(780 \mathrm{~cm}^{-1}\right)$ and the strong asymmetric and symmetric stretching vibration modes of $\mathrm{CH}_{2}$ at 2923 and 2854 $\mathrm{cm}^{-1}$, respectively. They also present $-\mathrm{NH}\left(1579 \mathrm{~cm}^{-1}\right), \mathrm{C}-\mathrm{N}$ $\left(1490 \mathrm{~cm}^{-1}\right)$, and $\mathrm{C}-\mathrm{N}\left(1315 \mathrm{~cm}^{-1}\right)$ bands, which confirms the presence of APTES on the surface of perovskite NCs. ${ }^{44}$ The absence of the $\mathrm{NH}_{2}$ stretching band $\left(3300-3400 \mathrm{~cm}^{-1}\right)$ could be attributed to hydrogen bindings that result in a peak broadening in the infrared spectrum of a molecule. On the other hand, core-shell nanoparticles functionalized with vinyltriethoxysilane exhibit the characteristic features of the vinyl group (i.e., $\nu_{\mathrm{st}}(\mathrm{C}=\mathrm{C}): 1629 \mathrm{~cm}^{-1} ; \delta\left(\mathrm{CH}_{2}=\mathrm{CH}\right): 1503 \mathrm{~cm}^{-1}$; $\left.\delta\left(=\mathrm{CH}_{2}\right): 1382 \mathrm{~cm}^{-1}\right) .{ }^{45}$ These results support the versatility of our NCs, whose surface chemistry could be easily adjusted by post-synthetic treatment with adequate alkoxysilane.

Finally, as a proof of concept, the capability for immunosensing of fluorogenic labels based on the developed MHP NCs conjugated to the IgG antibody has been proven by a direct immunoassay onto polycarbonate cuvettes, Figure 6a. For this purpose, core-shell NPs were conjugated to the anti-BSA antibody by passive adsorption. In parallel, polycarbonate (PC) cuvettes were incubated overnight with the BSA protein in buffer saline phosphate (PBS, $\mathrm{pH}=7$ ). After washing, the NPanti-BSA antibody conjugate was added in cuvettes and incubated for $2 \mathrm{~h}$. Finally, PL was recorded (for further experimental details see the Supporting Information).

In Figure $6 \mathrm{~b}, \mathrm{c}$ are depicted the PL response and signal to noise ratio for cuvettes treated with anti-BSA-NP conjugates at different concentrations. It is noteworthy that these results confirm the successful biorecognition of the target, achieving a signal to noise ratio of 25. Moreover, a control experiment where BSA-coated cuvettes were treated with NPs in the absence of antibodies presents a negligible PL response. The lower signal to noise ratio registered for samples treated with a higher antibody concentration could be attributed to the large antibody/ nanoparticle ratio, which may lead to a high antibody loading and a reduction of the immunoreactivity because of the steric hindrance. $^{46,47}$ Although additional research needs to be done, such as the attainment of MHP core-shell NCs that preserves their native PL yield and the optimization of antibody/ nanoparticle ratio, our work goes one step forward and demonstrates the feasibility and potential of perovskite nanoparticles as a labeling tool in immunochemistry.

A comparison of our work with the current state of the art is depicted in Table S4 (Supporting Information). Remarkably, there are several works which report the encapsulation of $\mathrm{CsPbBr}_{3} \mathrm{NCs}$ into an oxide matrix at a single-particle level. Considering the requirements of luminescent nanoparticles as a fluorescent label, nanoparticles with an average diameter between 1 and $100 \mathrm{~nm}$ present dimensions comparable to biological functional units and have been demonstrated to be effective for biosensing and bioimaging applications. ${ }^{48}$ In addition, they should be also monodisperse, stable in an aqueous medium, and preferably spherical because of the more efficient immobilization of biomolecules on the nanoparticle surface without variations in curvature. ${ }^{49}$ Among the different works in the literature, there are only three reports that accomplish all requirements. On the one hand, Zhong et al. described quasi-spherical $\mathrm{CsPbBr}_{3} @ \mathrm{SiO}_{2}$ core-shell NPs prepared via a supersaturated recrystallization method in which perovskite precursors were injected into a bad solvent (toluene) containing the alkoxysilane solution. ${ }^{18}$ Song et al. ${ }^{9}$ synthesized core-shell cube-shaped nanoparticles via a modified hot injection method, wherein oleylamine was partially substituted with APTES and Huang et al. prepared core-shell NPs via in situ growth into a hollow siliceous nanosphere template. ${ }^{11}$

In this sense, our work constitutes an alternative approach to the existing field for controlled synthesis of monodispersed core-shell NCs with suitable properties to be used as a fluorogenic label based on the post-synthetic treatment of assynthesized $\mathrm{CsPbBr}_{3} \mathrm{NCs}$ with TEOS and aqueous ammonia. This work sheds light on the chemical processes involved in the silica nucleation mechanism in the presence of perovskite nanoparticles and the role of ammonia and fractioned water addition in the phase transformation and the formation of spherical and monodisperse $\mathrm{CsPb}_{2} \mathrm{Br}_{5} @ \mathrm{SiO}_{2}$ core-shell NPs. Although we are fully aware that the emission efficiency of our developed NPs is too low and further investigation is mandatory to prepare NPs which maintain their native structure and PLQY intact, for instance, the study of a larger basic catalyst, which does not penetrate into a crystal network or the introduction of pre-passivation steps of $\mathrm{CsPbBr} \mathrm{Pb}_{3} \mathrm{NCs}$.

With respect to applications, Song et al. ${ }^{9}$ and Ding et al. ${ }^{20}$ employed their developed NPs for cell imaging in tumoral culture. In this sense, our work makes the difference because we develop a fluorescent specific label via the formation of antibody-conjugated nanoparticles for specific protein detection. Thus, our developed NPs can combine the small size and benefits of perovskite nanoparticles with the abilities of antibodies for specific recognition of a selected target. An accurate analysis of biomarker molecules is essential for the early detection, treatment, and management of diseases. This implies applications for diagnosis by immunoassay in cell or immunostaining or their use as a multimodal fluorescent contrast agent in cell tracking, transfection, and so forth.

\section{CONCLUSIONS}

In summary, in this work we have demonstrated the synthesis of spherical blue luminescent monodisperse $\mathrm{CsPb}_{2} \mathrm{Br}_{5} @ \mathrm{SiO}_{2}$ core-shell NPs, fluorescent labels for biosensing, via a ligandmediated transformation of pre-synthesized $\mathrm{CsPbBr}_{3} \mathrm{NCs}$ in the presence of ammonia, water, and TEOS. The easy and effective functionalization of the developed nanoparticles with amine and vinyl groups by additional post-synthetic treatment with corresponding alkoxysilanes was confirmed by ATR-FTIR spectroscopy. Finally, going one step further, we demonstrated the application of the developed core-shell NCs as luminescent for biosensing by QD-labeled anti-BSA antibody fluorescence immunoassays, which were applied to the specific detection of the BSA protein.

Our work provides insights into $\mathrm{CsPbX}_{3}$ phase transformations in the presence of TEOS, water, and ammonia, thereby improving the fundamental understanding of the underlying silica growth chemistry and informing future synthetic and post-synthetic efforts toward the design of robust and efficient $\mathrm{MHP} @ \mathrm{SiO}_{2}$ core-shell nanoparticles with the desired surface functionality and with potential to work as fluorescent labels for bioimaging, biosensing, and molecular detection. 


\section{ASSOCIATED CONTENT}

\section{(s) Supporting Information}

The Supporting Information is available free of charge at https://pubs.acs.org/doi/10.1021/acsanm.0c03340.

Experimental section; description of as-synthesized green emitting $\mathrm{CsPbBr}_{3}$ NCs optical and structural features; TEM images of $\mathrm{CsPbBr}_{3} \mathrm{NCs}$ treated with different amounts TEOS and aqueous ammonia and control samples; elemental mapping images of samples B and $\mathrm{G}$; evolution of PL intensity of core-shell nanoparticles in water solvent and change in relative fluorescence intensity as a function of time; HR-TEM image of $\mathrm{CsPb}_{2} \mathrm{Br}_{5} @ \mathrm{SiO}_{2}$ $\mathrm{NCs}$, table summary of structural features and reactant concentrations for the different synthesized $\mathrm{SiO}_{2}$-overcoated perovskite NPs; element quantification obtained from ICP-MS; and summary of the state of the art for the synthesis of $\mathrm{MHP} @ \mathrm{SiO}_{2}$ core-shell nanoparticles (PDF)

\section{AUTHOR INFORMATION}

\section{Corresponding Author}

Victoria González Pedro - Instituto Interuniversitario de Investigación de Reconocimiento Molecular y Desarrollo Tecnológico (IDM), Universitat Politècnica de ValènciaUniversitat de València, E46022 València, Spain; ๑ orcid.org/0000-0002-8133-5958; Email: vigonpe@ upvnet.upv.es

\section{Authors}

Cynthia Collantes - Instituto Interuniversitario de Investigación de Reconocimiento Molecular y Desarrollo Tecnológico (IDM), Universitat Politècnica de ValènciaUniversitat de València, E46022 València, Spain

María-José Bañuls - Instituto Interuniversitario de Investigación de Reconocimiento Molecular y Desarrollo Tecnológico (IDM), Universitat Politècnica de ValènciaUniversitat de València, E46022 València, Spain; Departamento de Química, Universitat Politècnica de València, E46022 València, Spain; (1) orcid.org/0000-00022422-7731

Ángel Maquieira - Instituto Interuniversitario de Investigación de Reconocimiento Molecular y Desarrollo Tecnológico (IDM), Universitat Politècnica de València-Universitat de València, E46022 València, Spain; Departamento de Química, Universitat Politècnica de València, E46022 València, Spain; (1) orcid.org/0000-0003-4641-4957

Complete contact information is available at:

https://pubs.acs.org/10.1021/acsanm.0c03340

\section{Author Contributions}

C.C. and V.G.P. designed the methodology and performed the data curation and formal analysis. V.G.P. and M.J.-B. conceptualized the investigation and A.M. supervised this study. V.G.P. wrote the first version of the manuscript. All authors participated in the investigation and data discussion and contributed to paper reviewing and editing.

\section{Notes}

The authors declare no competing financial interest.

\section{ACKNOWLEDGMENTS}

This work was financially supported by the E.U FEDER projects BiHolog CTQ2016-75749-R and ADBIHOL (PID2019110713RB-I00) from MINECO and PROMETEO/2020/094 from Generalitat Valenciana. C.C. thanks the Spanish Ministry of Economy and Competitiveness for her predoctoral contract (FPI/17 Scholarship). V.G-P. thanks Universitat Politècnica de València for her postdoctoral fellowship (Grant-PAID-10-14) and for financial support from the project PAID/16/18 (VIITUPV).

\section{REFERENCES}

(1) Quan, L. N.; Rand, B. P.; Friend, R. H.; Mhaisalkar, S. G.; Lee, T. W.; Sargent, E. H. Perovskites for Next-Generation Optical Sources. Chemical Reviews 2019, 7444-7477.

(2) Zheng, W.; Huang, P.; Gong, Z.; Tu, D.; Xu, J.; Zou, Q.; Li, R.; You, W.; Bünzli, J.-C. G.; Chen, X. Near-Infrared-Triggered Photon Upconversion Tuning in All-Inorganic Cesium Lead Halide Perovskite Quantum Dots. Nat. Commun. 2018, 9, 3462.

(3) Manzi, A.; Tong, Y.; Feucht, J.; Yao, E.-P.; Polavarapu, L.; Urban, A. S.; Feldmann, J. Resonantly Enhanced Multiple Exciton Generation through Below-Band-Gap Multi-Photon Absorption in Perovskite Nanocrystals. Nat. Commun. 2018, 9, 1518.

(4) Chen, W.; Bhaumik, S.; Veldhuis, S. A.; Xing, G.; Xu, Q.; Grätzel, M.; Mhaisalkar, S.; Mathews, N.; Sum, T. C. Giant Five-Photon Absorption from Multidimensional Core-Shell Halide Perovskite Colloidal Nanocrystals. Nat. Commun. 2017, 8, 15198.

(5) Tenne, R.; Rossman, U.; Rephael, B.; Israel, Y.; Krupinski-Ptaszek, A.; Lapkiewicz, R.; Silberberg, Y.; Oron, D. Super-Resolution Enhancement by Quantum Image Scanning Microscopy. Nat. Photonics 2019, 13, 116-122.

(6) Guerrero-Martínez, A.; Pérez-Juste, J.; Liz-Marzán, L. M. Recent Progress on Silica Coating of Nanoparticles and Related Nanomaterials. Adv. Mater. 2010, 22, 1182-1195.

(7) Li, Z.; Kong, L.; Huang, S.; Li, L. Highly Luminescent and Ultrastable $\mathrm{Cs} \mathrm{PbBr} 3$ Perovskite Quantum Dots Incorporated into a Silica/Alumina Monolith. Angew. Chem. Int. Ed. 2017, 56, 8134-8138.

(8) Huang, H.; Chen, B.; Wang, Z.; Hung, T. F.; Susha, A. S.; Zhong, H.; Rogach, A. L. Water resistant $\mathrm{CsPbX} 3$ nanocrystals coated with polyhedral oligomeric silsesquioxane and their use as solid state luminophores in all-perovskite white light-emitting devices. Chem. Sci. 2016, 7, 5699-5703.

(9) Song, W.; Wang, Y.; Wang, B.; Yao, Y.; Wang, W.; Wu, J.; Shen, Q.; Luo, W.; Zou, Z. Super stable CsPbBr3@SiO2 tumor imaging reagent by stress-response encapsulation. Nano Res. 2020, 13, 795-801.

(10) Huang, S.; Li, Z.; Kong, L.; Zhu, N.; Shan, A.; Li, L. Enhancing the Stability of $\mathrm{CH} 3 \mathrm{NH} 3 \mathrm{PbBr} 3$ Quantum Dots by Embedding in Silica Spheres Derived from Tetramethyl Orthosilicate in "Waterless" Toluene. J. Am. Chem. Soc. 2016, 138, 5749-5752.

(11) Huang, Y.; Li, F.; Qiu, L.; Lin, F.; Lai, Z.; Wang, S.; Lin, L.; Zhu, Y.; Wang, Y.; Jiang, Y.; Chen, X. Enhancing the Stability of $\mathrm{CH} 3 \mathrm{NH} 3 \mathrm{PbBr} 3 \mathrm{Nanoparticles} \mathrm{Using} \mathrm{Double} \mathrm{Hydrophobic} \mathrm{Shells} \mathrm{of}$ $\mathrm{SiO} 2$ and Poly(vinylidene fluoride). ACS Appl. Mater. Interfaces 2019, 11, 26384-26391.

(12) Cai, J.; Gu, K.; Zhu, Y.; Zhu, J.; Wang, Y.; Shen, J.; Trinchi, A.; Li, C.; Wei, G. Highly stable CsPbBr3@SiO2 nanocomposites prepared via confined condensation for use as a luminescent ink. Chem. Commun. 2018, 54, 8064-8067.

(13) Sun, C.; Zhang, Y.; Ruan, C.; Yin, C.; Wang, X.; Wang, Y.; Yu, W. W. Efficient and Stable White LEDs with Silica-Coated Inorganic Perovskite Quantum Dots. Adv. Mater. 2016, 28, 10088-10094.

(14) Malgras, V.; Tominaka, S.; Ryan, J. W.; Henzie, J.; Takei, T.; Ohara, K.; Yamauchi, Y. Observation of Quantum Confinement in Monodisperse Methylammonium Lead Halide Perovskite Nanocrystals Embedded in Mesoporous Silica. J. Am. Chem. Soc. 2016, 138, 1387413881.

(15) González-Pedro, V.; Veldhuis, S. A.; Begum, R.; Bañuls, M. J.; Bruno, A.; Mathews, N.; Mhaisalkar, S.; Maquieira, A. Recovery of Shallow Charge-Trapping Defects in CsPbX3 Nanocrystals through Specific Binding and Encapsulation with Amino-Functionalized Silanes. ACS Energy Lett. 2018, 3, 1409-1414. 
(16) Luo, B.; Pu, Y.-C.; Lindley, S. A.; Yang, Y.; Lu, L.; Li, Y.; Li, X.; Zhang, J. Z. Organolead Halide Perovskite Nanocrystals: Branched Capping Ligands Control Crystal Size and Stability. Angew. Chem. Int. Ed. 2016, 55, 8864-8868.

(17) Hu, H.; Wu, L.; Tan, Y.; Zhong, Q.; Chen, M.; Qiu, Y.; Yang, D.; Sun, B.; Zhang, Q.; Yin, Y. Interfacial Synthesis of Highly Stable $\mathrm{CsPbX3/Oxide} \mathrm{Janus} \mathrm{Nanoparticles.} \mathrm{J.} \mathrm{Am.} \mathrm{Chem.} \mathrm{Soc.} \mathrm{2018,} \mathrm{140,} \mathrm{406-}$ 412.

(18) Zhong, Q.; Cao, M.; Hu, H.; Yang, D.; Chen, M.; Li, P.; Wu, L.; Zhang, Q. One-Pot Synthesis of Highly Stable CsPbBr3@SiO2 CoreShell Nanoparticles. ACS Nano 2018, 12, 8579-8587.

(19) Cheng, J.; Yuan, S.; Zhu, L.; Chen, L.; Liu, C.; Tong, H.; Zeng, H. Room-Temperature in Situ Synthesis of Highly Efficient CsPbBr3/ $\mathrm{SiO} 2 \mathrm{Sol}$ in Entirely Ethanol Solvent by Constructing AmineFunctionalized Silica Micelles. Langmuir 2020, 36, 3565-3572.

(20) Ding, N.; Zhou, D.; Sun, X.; Xu, W.; Xu, H.; Pan, G.; Li, D.; Zhang, S.; Dong, B.; Song, H. Highly stable and water-soluble monodisperse $\mathrm{CsPbX} 3 / \mathrm{SiO} 2$ nanocomposites for white- $\mathrm{LED}$ and cells imaging. Nanotechnology 2018, 29, 345703.

(21) Wei, Y.; Cheng, Z.; Lin, J. An Overview on Enhancing the Stability of Lead Halide Perovskite Quantum Dots and Their Applications in Phosphor-Converted LEDs. Chem. Soc. Rev. 2019, 310-350.

(22) Protesescu, L.; Yakunin, S.; Bodnarchuk, M. I.; Krieg, F.; Caputo, R.; Hendon, C. H.; Yang, R. X.; Walsh, A.; Kovalenko, M. V. Nanocrystals of Cesium Lead Halide Perovskites (CsPbX3, X $=\mathrm{Cl}, \mathrm{Br}$, and I): Novel Optoelectronic Materials Showing Bright Emission with Wide Color Gamut. Nano Lett. 2015, 15, 3692-3696.

(23) Wang, J.; Sugawara-Narutaki, A.; Fukao, M.; Yokoi, T.; Shimojima, A.; Okubo, T. Two-Phase Synthesis of Monodisperse Silica Nanospheres with Amines or Ammonia Catalyst and Their Controlled Self-Assembly. ACS Appl. Mater. Interfaces 2011, 3, 15381544.

(24) Li, P.; Yang, D.; Tan, Y.; Cao, M.; Zhong, Q.; Chen, M.; Hu, H.; Sun, B.; Xu, Y.; Zhang, Q. Consecutive Interfacial Transformation of Cesium Lead Halide Nanocubes to Ultrathin Nanowires with Improved Stability. ACS Appl. Mater. Interfaces 2019, 11, 3351-3359. (25) Liu, Z.; Bekenstein, Y.; Ye, X.; Nguyen, S. C.; Swabeck, J.; Zhang, D.; Lee, S.-T.; Yang, P.; Ma, W.; Alivisatos, A. P. Ligand Mediated Transformation of Cesium Lead Bromide Perovskite Nanocrystals to Lead Depleted Cs4PbBr6 Nanocrystals. J. Am. Chem. Soc. 2017, 139, 5309-5312.

(26) Hintermayr, V. A.; Richter, A. F.; Ehrat, F.; Döblinger, M.; Vanderlinden, W.; Sichert, J. A.; Tong, Y.; Polavarapu, L.; Feldmann, J.; Urban, A. S. Tuning the Optical Properties of Perovskite Nanoplatelets through Composition and Thickness by Ligand-Assisted Exfoliation. Adv. Mater. 2016, 28, 9478-9485.

(27) Zhang, X.; Bai, X.; Wu, H.; Zhang, X.; Sun, C.; Zhang, Y.; Zhang, W.; Zheng, W.; Yu, W. W.; Rogach, A. L. Water-Assisted Size and Shape Control of $\mathrm{CsPbBr} 3$ Perovskite Nanocrystals. Angew. Chem. Int. Ed. 2018, 57, 3337-3342.

(28) Leguy, A. M. A.; Hu, Y.; Campoy-Quiles, M.; Alonso, M. I.; Weber, O. J.; Azarhoosh, P.; Van Schilfgaarde, M.; Weller, M. T.; Bein, T.; Nelson, J.; Docampo, P.; Barnes, P. R. F. Reversible Hydration of CH3NH3PbI3in Films, Single Crystals, and Solar Cells. Chem. Mater. 2015, 27, 3397-3407.

(29) Lou, S.; Xuan, T.; Wang, J. (INVITED) Stability: A desiderated problem for the lead halide perovskites. Opt. Mater.: X 2019, 1, 100023.

(30) Dursun, I.; De Bastiani, M.; Turedi, B.; Alamer, B.; Shkurenko, A.; Yin, J.; El-Zohry, A. M.; Gereige, I.; AlSaggaf, A.; Mohammed, O. F.; Eddaoudi, M.; Bakr, O. M. CsPb2Br5Single Crystals: Synthesis and Characterization. ChemSusChem 2017, 10, 3746-3749.

(31) Li, G.; Wang, H.; Zhu, Z.; Chang, Y.; Zhang, T.; Song, Z.; Jiang, $\mathrm{Y}$. Shape and phase evolution from $\mathrm{CsPbBr} 3$ perovskite nanocubes to tetragonal $\mathrm{Cs} \mathrm{Pb} 2 \mathrm{Br} 5$ nanosheets with an indirect bandgap. Chem. Commun. 2016, 52, 11296-11299.

(32) Li, J.; Zhang, H.; Wang, S.; Long, D.; Li, M.; Guo, Y.; Zhong, Z.; $\mathrm{Wu}, \mathrm{K}$.; Wang, D.; Zhang, T. Synthesis of all-inorganic CsPb2Br5 perovskite and determination of its luminescence mechanism. RSC Adv. 2017, 7, 54002-54007.

(33) Balakrishnan, S. K.; Kamat, P. V. Ligand Assisted Transformation of Cubic CsPbBr3 Nanocrystals into Two-Dimensional CsPb2Br5 Nanosheets. Chem. Mater. 2018, 30, 74-78.

(34) Wang, K.-H.; Wu, L.; Li, L.; Yao, H.-B.; Qian, H.-S.; Yu, S.-H. Large-Scale Synthesis of Highly Luminescent Perovskite-Related $\mathrm{CsPb} 2 \mathrm{Br} 5$ Nanoplatelets and Their Fast Anion Exchange. Angew. Chem. 2016, 128, 8468-8472.

(35) Tang, X.; Hu, Z.; Yuan, W.; Hu, W.; Shao, H.; Han, D.; Zheng, J.; Hao, J.; Zang, Z.; Du, J.; Leng, Y.; Fang, L.; Zhou, M. Perovskite $\mathrm{Cs} \mathrm{Pb} 2 \mathrm{Br} 5$ Microplate Laser with Enhanced Stability and Tunable Properties. Adv. Opt. Mater. 2017, 5, 1600788.

(36) Han, C.; Li, C.; Zang, Z.; Wang, M.; Sun, K.; Tang, X.; Du, J. Tunable luminescent $\mathrm{CsPb} 2 \mathrm{Br} \_5$ nanoplatelets: applications in lightemitting diodes and photodetectors. Photon. Res. 2017, 5, 473.

(37) Zhang, Y.; Yin, J.; Parida, M. R.; Ahmed, G. H.; Pan, J.; Bakr, O. M.; Brédas, J.-L.; Mohammed, O. F. Direct-Indirect Nature of the Bandgap in Lead-Free Perovskite Nanocrystals. J. Phys. Chem. Lett. 2017, 8, 3173-3177.

(38) May, T. W.; Wiedmeyer, R. H. A Table of Polyatomic Interferences in ICP-MS. At. Spectrosc. 1998, 19, 150-155.

(39) Horlick, G.; Tan, S. H. Background Spectral Features in Inductively Coupled Plasma/Mass Spectrometry. Appl. Spectrosc. 1986, 40, 445-460.

(40) Maes, J.; Balcaen, L.; Drijvers, E.; Zhao, Q.; De Roo, J.; Vantomme, A.; Vanhaecke, F.; Geiregat, P.; Hens, Z. Light Absorption Coefficient of $\mathrm{CsPbBr} 3$ Perovskite Nanocrystals. J. Phys. Chem. Lett. 2018, 9, 3093-3097.

(41) Liu, M.; Zhao, J.; Luo, Z.; Sun, Z.; Pan, N.; Ding, H.; Wang, X. Unveiling Solvent-Related Effect on Phase Transformations in CsBr$\mathrm{PbBr} 2$ System: Coordination and Ratio of Precursors. Chem. Mater. 2018, 30, 5846-5852.

(42) Lamer, V. K.; Dinegar, R. H. Theory, Production and Mechanism of Formation of Monodispersed Hydrosols. J. Am. Chem. Soc. 1950, 72, 4847-4854.

(43) Ding, H. L.; Zhang, Y. X.; Wang, S.; Xu, J. M.; Xu, S. C.; Li, G. H. Fe3O4@SiO2 Core/Shell Nanoparticles: The Silica Coating Regulations with a Single Core for Different Core Sizes and Shell Thicknesses. Chem. Mater. 2012, 24, 4572-4580.

(44) Aneja, K. S.; Bohm, S.; Khanna, A. S.; Bohm, H. L. M. Graphene Based Anticorrosive Coatings for $\mathrm{Cr}(\mathrm{VI})$ Replacement. Nanoscale 2015, 7, 17879-17888.

(45) Peng, S.; Zeng, Z.; Zhao, W.; Li, H.; Chen, J.; Han, J.; Wu, X. Novel Functional Hybrid Silica Sol-Gel Coating for Copper Protection via in Situ Thiol-Ene Click Reaction. RSC Adv. 2014, 4, 15776-15781.

(46) Tripathi, K.; Driskell, J. D. Quantifying Bound and Active Antibodies Conjugated to Gold Nanoparticles: A Comprehensive and Robust Approach to Evaluate Immobilization Chemistry. ACS Omega 2018, 3, 8253-8259.

(47) Dobosz, P.; Morais, S.; Puchades, R.; Maquieira, A. Nanogold Bioconjugates for Direct and Sensitive Multiplexed Immunosensing. Biosens. Bioelectron. 2015, 69, 294-300.

(48) Nune, S. K.; Gunda, P.; Thallapally, P. K.; Lin, Y.-Y.; Laird Forrest, M.; Berkland, C. J. Nanoparticles for biomedical imaging. Expet Opin. Drug Deliv. 2009, 6, 1175-1194.

(49) Byzova, N. A.; Zherdev, A. V.; Khlebtsov, B. N.; Burov, A. M.; Khlebtsov, N. G.; Dzantiev, B. B. Advantages of Highly Spherical Gold Nanoparticles as Labels for Lateral Flow Immunoassay. Sensors 2020, 20, 3608-15. 